\title{
Trends in 24-hour urine protein in pregestational diabetics as predictors for the development of pregnancy related hypertensive disorders
}

\author{
Kendra M GRAY ${ }^{1 *}$, Rachel L RODEL ${ }^{1,2}$, Richard GERKIN ${ }^{1}$, Ana BODEA BRAESCU ${ }^{3}$ and Christopher Kevin HULS ${ }^{1,3}$ \\ ${ }^{1}$ University of Arizona College of Medicine Phoenix at Banner University Medical Center, Obstetrics and Gynecology Department, Arizona, USA \\ ${ }^{2}$ University of Colorado Denver (Current), Obstetrics and Gynecology Department, Aurora, USA \\ ${ }^{3}$ Mednax Medical Group, Arizona, USA
}

\begin{abstract}
Objective(s): To establish a trend in the physiologic changes in urine protein collected over a 24-hour period throughout the course of pregnancy in women with pregestational diabetes. To determine that the risk for developing preeclampsia can be predicted by 24-hr urine protein in the first trimester and validate first trimester 24-hour urine protein in predicting neonatal outcomes.
\end{abstract}

Study Design: This was a retrospective cohort study of 150 women with pregestational diabetes who underwent multiple 24 -hour urine protein collections spanning at least two trimesters and the outcomes of their infants.

Main Outcome Measures: Differences in first and third trimester proteinuria results were analyzed. Marginal means was used to establish a change in 24-hour urine excretion over the course of multiple trimesters for eligible data points. Regression analysis and locally weighted scatterplot smoothing was performed to illustrate a trend. Correlational testing between first trimester 24-hour urine protein and neonatal outcomes was analyzed.

Results: Distribution of 24-hour urine protein levels throughout pregnancy was demonstrated. The mean 24-hour urine protein in women without the diagnosis of gestational hypertension or preeclampsia was $361 \mathrm{mg}$ (SD 542.00), median 148.00. Women who received a diagnosis had mean third trimester 24-hour urine protein value of $2178.50 \mathrm{mg}$ (SD 3173.20) with a median $8120.50 \mathrm{mg}$. This difference was statistically significant $(\mathrm{p}=0.01)$.

Conclusion(s): A value of $450 \mathrm{mg}$ would have an $85 \%$ specificity and nearly $70 \%$ sensitivity for predicting preeclampsia. First trimester $24-$ hour urine protein is a poor predictor of gestational hypertension and preeclampsia.

\section{Introduction}

More than 8 million women in the United States have pregestational diabetes mellitus, and it is observed in $1 \%$ of all pregnancies [1]. A $24-$ hour urine collection for protein excretion is advised at preconception counseling and as part of early routine prenatal care for the gravida with pregestational diabetes, however, its clinical utility has not been fully established. Exceptions to the statement include cases of severe nephropathy where pregnancy may be outright discouraged [2]. Repeat testing is advised based on provider discretion and clinical scenario, although indications are ambiguous. It is generally accepted that women have worsening physiologic proteinuria in the third trimester of pregnancy. Up to a $300 \mathrm{mg}$ per day threshold is considered physiologic in pregnancy in non-diabetic women [3]. The difficulty arises when a woman with pregestational diabetes, but without evidence of microalbuminuria before pregnancy, develops an exaggerated pregnancy induced protein excretion that exceeds the acceptable $300 \mathrm{mg}$ per day. This is of interest clinically because it substantially complicates the diagnosis of preeclampsia and related disorders.

It is well-established that women with pregestational diabetes are at an increased risk of complications during pregnancy, including preeclampsia [4]. Though not required, worsening of urine protein throughout pregnancy has the potential to be interpreted as end-organ damage and used as inclusion criterion for the diagnosis of preeclampsia
[5]. This has the potential to impact the decision of timing of delivery or may result in further interventions such as inpatient admission, increased fetal monitoring, invasive testing and administration of medications such as magnesium sulfate or steroids when interventions may not be warranted. One study of 462 women with pregestational diabetes showed that roughly $20 \%$ developed preeclampsia [6]. That study also demonstrated that those patients with baseline proteinuria had further increased risk of developing preeclampsia with their rate found to be $28 \%$. There is not a commonly accepted definition for proteinuria for patients with pregestational diabetes and literature previously demonstrating the physiologic changes in this population is lacking. There is no well-established threshold for quantity of urine protein over a 24 -hour period at baseline in early pregnancy to predict preeclampsia. There is also no well-established threshold for quantity of urine protein over a 24 -hour period at baseline in early pregnancy

*Correspondence to: Kendra M Gray, PO Box 27582, Scottsdale, AZ 85255, USA, Tel: (480) 272-3555 (M), (602) 839-3827 (O); Fax: (480) 522-1951; E-mail: kendramgray@gmail.com

Key words: 24-hour urine protein, hypertensive disorders in pregnancy, pregestational diabetes

Received: June 05, 2018; Accepted: June 26, 2018; Published: June 28, 2018 
for predicting preeclampsia in the third trimester. Furthermore, no established increase above baseline in urine protein over the course of a woman's pregnancy has been defined as pathological.

Our objective is to establish a trend in the physiologic changes in urine protein collected over a 24 -hour period in pregestational diabetics throughout gestation. We hypothesize that the risk for developing preeclampsia can be predicted by 24 -hour urine protein in the first trimester and that this would establish its utility in differentiating between preeclampsia, nephropathy and physiologic changes later in pregnancy. We further believe the first trimester 24-hour urine protein will aid in predicting neonatal outcomes of Apgar scores, birthweight and timing of delivery.

\section{Methods}

\section{Human subjects review and approval}

Approval was obtained from the Banner University Medical Center Phoenix IRB under the title "Retrospective Analysis of Changes in 24hour Urine Protein Throughout Pregnancy in Pregestational Diabetics." Informed consent was not required for study purposes as this was a retrospective chart review. Informed consent for testing was given at the time of laboratory collection.

\section{Study design}

A retrospective cohort study was performed using charts from January 1, 2008 to December 31, 2012. Charts from Banner Cerner, Banner Peribirth, and Phoenix Perinatal and Associates clinic were reviewed. Charts were selected for analysis from patients who were diagnosed with pregestational diabetes and had two or more 24-hour urine protein collections recorded during pregnancy. No participants were excluded due to age, race, religion, employment, or ethnicity.

Three-hundred charts were analyzed. The primary objective was to establish a trend in 24-hour urine protein values over the course of pregnancy in pregestational diabetics. Pregestational diabetes was defined as Type I or Type II DM that was diagnosed before conception. Women that likely had undiagnosed pregestational diabetes suggested by elevated hemoglobin A1c levels early in pregnancy were excluded from analysis. Secondary objectives were aimed at investigating a correlation between first trimester 24-hour urine protein and the development of gestational hypertension, preeclampsia, hemoglobin Alc, patient body mass index, pregnancy weight gain, timing of delivery and short term neonatal outcomes including Apgar scores at one and five minutes and birth weight.

The charts were de-identified and given study identification numbers. Data collected included the patient's demographics, information regarding her obstetrical and past medical history including hemoglobin A1c values, age at diagnosis of diabetes mellitus, duration of diabetes mellitus, White's Classification, and therapy for her diabetes. Additional history regarding the presence and type of hypertension, presence of known renal disease and associated labs results were extracted. Outcome variable such as presence or absence of pregnancy related hypertensive disorders, gestational age at delivery, infant weight, and Apgar scores were recorded. IBM SPSS Statistics for Mac, version 24 (IBM Corp., Armonk, N.Y., USA) was used for the analysis.

\section{Statistical analysis}

For the primary objective, to trend the amount of urine protein in 24 hours throughout the course of pregnancy, charts with sufficient data extracted were included for analysis. To be eligible for analysis the patient needed to have at least one first trimester 24-hour urine and at least one additional 24-hour urine in the second or third trimester. Data was also included if the patient had her first 24-hour urine collection in the second trimester and a subsequent collection in the third trimester. Of the 300 charts available, one hundred and fifty $(\mathrm{N}=150)$ charts had useable data points. Some charts had multiple data points including first, second, and third trimester 24-hour urine protein collections. Other charts included first trimester and second trimester values only, second and third trimester values only or some combination thereof. Linear regression modeling and locally weighted scatterplot smoothing (LOESS) were used to trend changes in 24-hour urine protein through the first, second, and third trimester. Regression modeling was performed in one hundred and fifty $(n=150)$ of the 300 charts available that had data points to analyze. Baseline data is included in Table 1.

First trimester 24-hour urine protein values were also analyzed. Spearman's rho nonparametric testing was used to assess a correlation between proteinuria in the first trimester and pregnancy related hypertensive disorders (gestational hypertension and preeclampsia). Mann-Whitney testing was used to determine a difference in protein values between the two groups. Third trimester 24-hour urinary protein values were analyzed in a similar fashion. To determine a value that could be used to predictably diagnose preeclampsia, data was plotted on a receiver operating characteristic (ROC) curve. The best cutoff point was established by analyzing the ROC curves by determining the cutoff that provided the highest sum of sensitivity and specificity.

In relation to the secondary objectives, Pearson $r$ correlation was used to measures the strength of association between 24-hour urine protein in the first trimester with each of the following measures: the development of preeclampsia, hemoglobin A1c, body mass index, weight gain during pregnancy, timing of delivery, and neonatal outcomes Apgar scoring, and birthweight. A two-tailed $\mathrm{p}<0.05$ was considered significant.

\section{Results}

Based on the data available, a trend of 24-hour urine protein through the course of gestation for women with pregestational diabetes was established using linear regression modeling and locally weighted scatterplot smoothing (LOESS). This is demonstrated visually as a display of the trend for the first, second, and third trimester values as shown in Figure 1.

In women with pregestational diabetes, there was no significant difference in first trimester 24-hour urine protein excretion in women without pregnancy related hypertensive disorders compared to women who received those diagnoses. The mean 24 -hour urine protein without diagnosis of pregnancy-related hypertensive disorders was $153.3 \mathrm{mg}$ (SD 157.3) with a median of 128.0. In women who ultimately received a diagnosis of gestational hypertension or preeclampsia the mean first trimester 24-hour urine protein was $287.5 \mathrm{mg}$ (SD 475.8) with a median $205.5 \mathrm{mg}$. This difference was not statistically significant $(\mathrm{p}=0.178)$.

Second trimester 24-hour urine proteins between these two groups demonstrated the mean 24-hour urine protein without diagnosis of pregnancy related hypertensive disorders was $139.8 \mathrm{mg}$ (SD 97.2), median 137.5. Women who received a diagnosis of gestational hypertension or preeclampsia had mean second trimester 24-hour urine protein values of $606.9 \mathrm{mg}$ (SD 2019.5) with a median $100.0 \mathrm{mg}$. This difference was not statistically significant $(\mathrm{p}=0.669)$.

Analysis of third trimester 24-hour urine proteins between these two groups demonstrated the mean 24-hour urine protein without 
Table 1. Patient Demographics

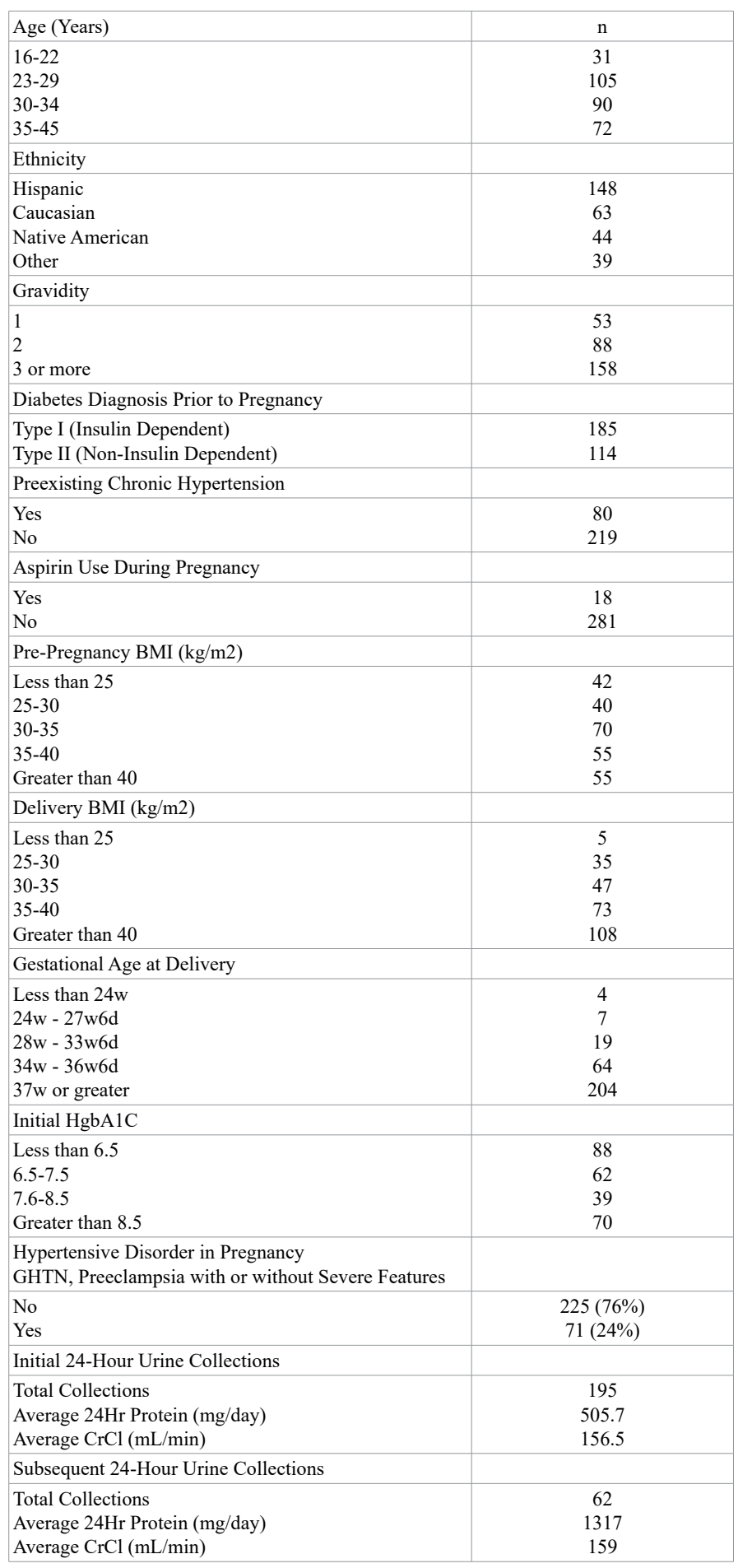

diagnosis of pregnancy related hypertensive disorders was $361 \mathrm{mg}(\mathrm{SD}$ 542.00), median 148.00. Women who received a diagnosis of gestational hypertension or preeclampsia had mean third trimester 24-hour urine protein values of $2178.50 \mathrm{mg}$ (SD 3173.20) with a median $8120.50 \mathrm{mg}$. This difference was statistically significant $(p=0.001)$. Assessment for the predictability of third trimester 24 -hour protein was analyzed and plotted on a ROC curve which had an area under the curve of 0.80 . The best cutoff point was established. At $450 \mathrm{mg}$ a 24 -hour urine protein collection in the third trimester is predictive of pregnancy related hypertensive disorders with a sensitivity of $69.4 \%$ and a specificity of 85\%. The ROC is displayed in Figure 2.

Secondary objectives were analyzed as previously described with the following results. Pearson $r$ correlation between 24-hour urine protein in the first trimester with first trimester hemoglobin Alc revealed no correlation $(\mathrm{r}=0.197, \mathrm{p}=0.167)$. There was insufficient data to evaluate the correlation between third trimester hemoglobin A1c and third trimester 24-hour urine protein. Spearmen's rho was used to analyze the remaining factors. There was no correlation between first trimester 24- hour urine protein and the development of preeclampsia ( $\rho=0.174, p=0.187)$, body mass index $\rho=0.165, p=0.211)$, weight gain during pregnancy $(\rho=0.082, p=0.562)$, timing of delivery $(\rho=0.212$, $p=0.107)$, and neonatal outcomes of one minute Apgar $(\rho=0.069, p=$ $0.603)$, five-minute Apgar $(\rho=0.115, p=0.390)$, and birthweight $(\rho=$ $-0.234, \mathrm{p}=0.071)$.

\section{Discussion}

The results of this study indicate that there appears to be a change in physiologic protein excretion in women with pregestational diabetes. This is demonstrated in a manner even more exaggerated than in pregnant nondiabetic counterparts. The trend in 24-hour urine protein through the various weeks of gestation appears to increase in an equivalent manner through the first trimester and increases at an accelerated rate in the second trimester in cases where pregnancy related hypertensive disorders are diagnosed. Due to changes in the software used for electronic medical records at the institution where the study took place, additional charts could not be accessed to establish a true nomogram which would be ideal.

The utility of first trimester 24-hour urinary protein testing based on this study has little value in predicting patients that develop pregnancy related hypertensive disorders. Prepregnancy 24-hour urine protein collections may be most useful in cases where macroproteinuria is discovered for identifying diabetic nephropathy. In those cases, it would be important to analyze the difference between preconception values and those in the first trimester. This allows providers and patients to discuss what risks, if any, pregnancy has on worsening renal function. This would allow for counseling regarding the risks of pregnancy based on underlying renal disease, serum creatinine, or the potential for changes during pregnancy. Counseling and evaluation in the prepregnancy period may allow for providers to initiate renal protective therapies such as ACE inhibitors immediately pospartum. ${ }^{5}$ In women without pregestational diabetes, measurement of protein excretion earlier in pregnancy has been proven in some studies to be predictive and thus education and initiation of prophylactic interventions such as aspirin and education regarding additional prenatal care can be initiated early on [7]. Women with pregestational diabetes, however, are already known to be at high risk for developing preeclampsia and other pregnancy complications and should be counseled regarding their risks early in pregnancy regardless of their 24-hour urine protein excretion levels $[8,9]$. The US Preventive Services Task Force has determined pregestational diabetes to be a high-risk group for development of preeclampsia and thus uniformly recommends administration of aspirin in pregnancy [10]. Given other risks associated with pregestational diabetes in pregnancy, women will likely already be undergoing further antenatal testing and collection of 24-hour urine during the first trimester, even though its value is unlikely to change clinical management [11]. Total urinary protein excretion in the first trimester is low and many not be warranted. 


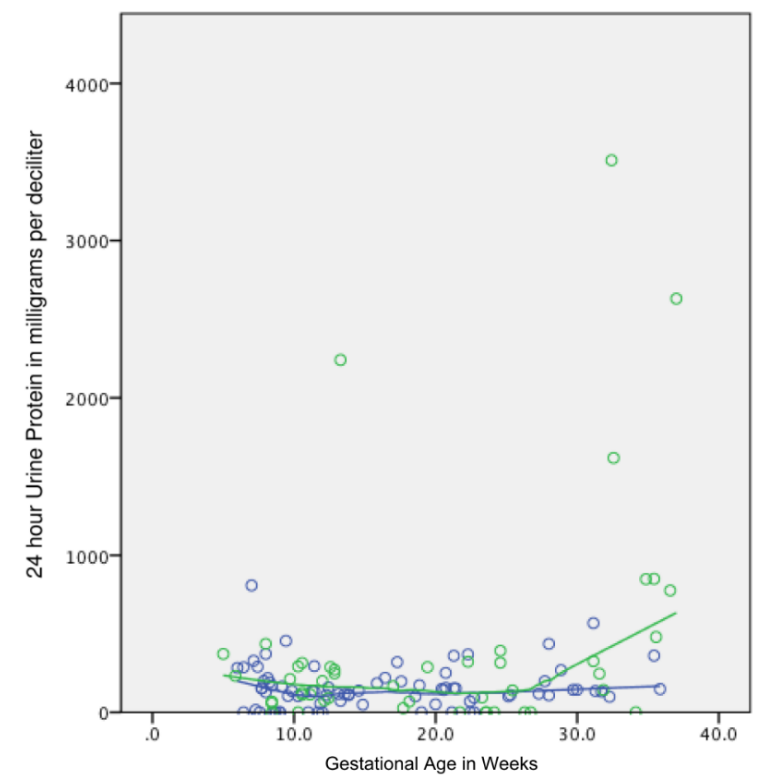

Pregestational Diabetic Women

Not Diagnosed with GHTN or Preeclampsia
Diagnosed with GHTN or Preeclampsia

Figure 1. Locally weighted scatterplot smoothing showing distribution of 24-hour urine protein levels throughout pregnancy

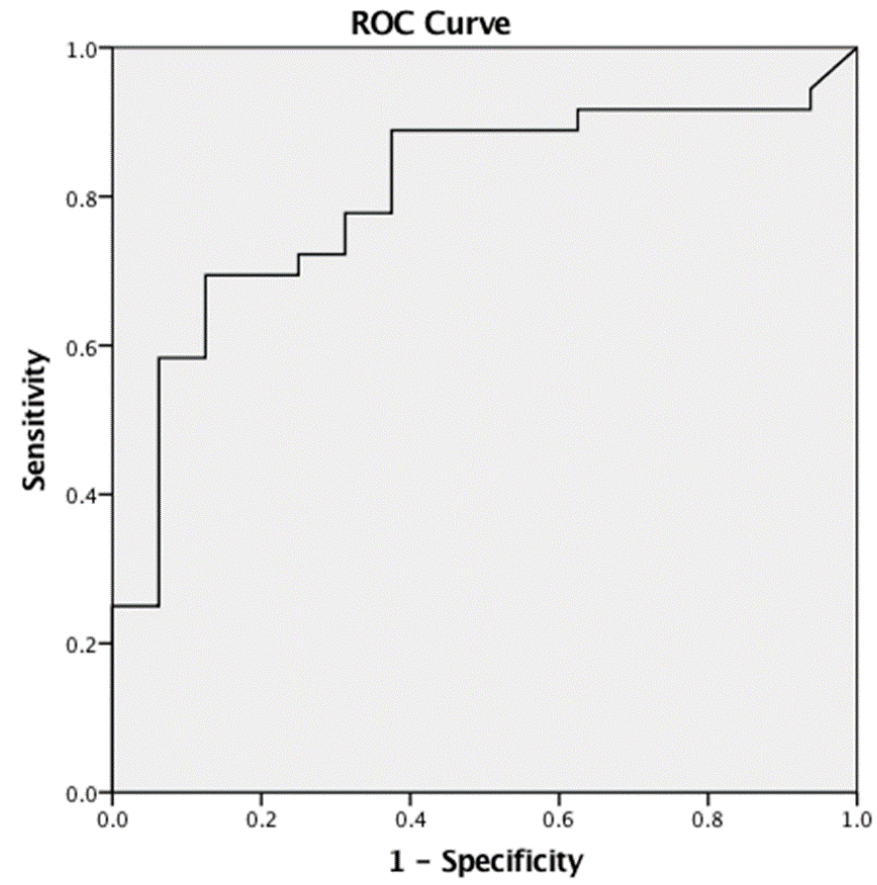

Diagonal segments are produced by ties.

Figure 2. Receiver Operating Characteristic Curve for Determining Sensitivity \& Specificity of 3rd Trimester 24-hour Urine Protein in Predicting Pregnancy Related Hypertensive Disorders. Area under the curve $=0.80$

Measurement of 24-hour urinary protein excretion has the benefits of being noninvasive and inexpensive, however, it is inconvenient [7]. Alternative testing with microalbuminuria or a protein to creatinine ratio may provide additional information without the inconvenience of a timed collection. Based on this study the utility of the 24-hour urinary protein excretion in predicting preeclampsia in the third trimester may be the most useful.

Of all the findings in this study, establishing a threshold 24-hour urine protein excretion level with high specificity for predicting preeclampsia in the third trimester may be the most useful. One might argue that a higher sensitivity test could be used to rule out cases of preeclampsia, however, given the physiologic worsening of 24-hour urine protein throughout pregnancy that is further exaggerated in the pregestational diabetic population, high specificity testing may be more beneficial. High specificity testing helps aid in the diagnosis of preeclampsia based on urine protein values, thereby minimizing the impact that confounding factors have in the diagnosis. As previously discussed, the diagnosis of preeclampsia in this population has been quite challenging [3]. Some literature and texts suggest the diagnosis of 
preeclampsia in a diabetic with no proteinuria baseline be made when the standard $300 \mathrm{mg}$ of protein is exceeded [5]. Based on our analysis, analysis, assuming all patients had no proteinuria at baseline, if the 24 $\mathrm{hr}$ urine protein values were $>300 \mathrm{mg}$ preeclampsia is predicted with a sensitivity of $=71 \%$ and specificity $84 \%$. Previous literature also comments that if the patient has proteinuria at baseline, exacerbation of proteinuria can be used in aiding the diagnosis, however again this is ill-defined [5]. We were unable to confirm or refute this statement. Our findings favor a higher threshold for diagnosis in this population as the mean proteinuria value in the third trimester in women not meeting criteria for diagnosis was $361 \mathrm{mg}$ (SD 542.00), well above the accepted physiological norm. There is a fine threshold between over diagnosis and unnecessary potentially harmful treatments weighed against the risk of excluding women from diagnosis and therefore restricting the benefits of treatment for both them and their fetuses. While this study cannot provide a conclusive threshold for the diagnosis of preeclampsia based on proteinuria in this population, a reasonable cutoff near 450 milligrams per deciliter in 24 hours is reasonable to consider as this gives a sensitivity of $69 \%$ and a specificity of $85 \%$.

Finally, evaluation of the secondary objectives assessing a correlation between first trimester 24-hour urine protein and the outcomes assessed including, hemoglobin A1c, body mass index, weight gain during pregnancy, timing of delivery, and neonatal outcomes of one and fiveminute Apgar, and birthweight continues to suggest the limited utility of this test in early pregnancy.

Limitations to the study include failure to examine 24-hour urine proteins at scheduled intervals or specific weeks of gestation during each of the trimesters to allow for more consistency in establishing a true nomogram between all subjects. The study is also limited by the fact that repeat 24-hour urine testing was ordered due to unknown clinical indications and at the discretion of the ordering provider. It would be reasonable to consider that symptomatology or blood pressure elevations were the catalyst for reassessment of 24-hour urine proteins after the first trimester. This has the potential to skew results toward an affiliation between third trimester urine proteins among patients in disease states versus a true sampling of women with pregestational diabetes. Further limitations include data collection from a single institution's population, potentially limiting generalizability. Both inhospital and outpatient labs were used to collect sample results which may contribute to interlaboratory variability. Data was collected at a time when the diagnosis of preeclampsia was made using alternate criteria which included the requirement of proteinuria. The best attempts were made to evaluate diagnosis based on current standards and those were used for analysis. Given the wide variability in the disease state of pregestational diabetes, there are multiple confounding factors that are difficult to control for, however this would be true even in a prospective study. In relation to secondary analyses, we cannot determine causation, only association. Additional limitations inherent to the nature of a retrospective cohort study include inferior level of evidence compared with prospective studies, smaller sample size, potential for convenience sampling and selection bias as alluded to earlier.

Beneficial future studies may include serial 24-hour urine protein collections at predetermined intervals throughout the pregnancy to establish a true nomogram. Ideally this should be stratified among diabetics without proteinuria prepregnancy, with microalbuminuria, and with macroalbuminuria complicating their condition. Other studies to consider include establishing a change or percentage above baseline that can be used for inclusion criteria for diagnosis of preeclampsia.

In conclusion, women with pregestational diabetes exhibit a statistically significant physiologic increase in urine protein throughout pregnancy. The difference in the increase over time between women with and without pregnancy related hypertensive disorders was found to be statistically significant in the third trimester. Values greater than $450 \mathrm{mg}$ have $85 \%$ specificity for predicting preeclampsia. First trimester 24-hour urine protein is a poor predictor of gestational hypertension, preeclampsia and outcomes such as development of gestational hypertension, development of preeclampsia, timing of delivery, one and five-minute Apgar scores, and neonatal birth weight. Its use in the diagnosis of gestational hypertension and preeclampsia should be utilized with caution. The only utility of an early 24-hour urine protein may be for those patients suspected of having or known to have nephropathy at baseline. Providers should be aware of the tests limitations in aiding or predicting the diagnosis of pregnancy related hypertensive disorders.

\section{Acknowledgment(s)}

Thanks to Meisje Burton, MD for her assistance with data collection.

\section{References}

1. Pregestational diabetes mellitus (2005) ACOG Practice Bulletin No. 60. American College of Obstetricians and Gynecologists. Obstet Gynecol 105: 675-685.

2. Preconception care of women with diabetes (2004) American Diabetes Association Diabetes Care 27 (suppl 1): S76-S78.

3. Creasy and Resnik's maternal-Fetal medicine: principles and practice. 7th ed Philadelphia, PA: Saunders / Elsevier; 2014.

4. Reece EA, Sivan E, Francis G, Homko CJ (1998) Pregnancy outcomes among women with and without microvascular disease (White's classes B to FR) versus non-diabetic controls. Am J Perinatol 15: 549-555. [Crossref]

5. The diabetes in pregnancy dilemma: leading change with proven solutions. Lanham University Press of America; 2006.

6. Sibai BM, Caritis S, Hauth J, Lindheimer M, VanDorsten JP, et al. (2000) Risks of preeclampsia and adverse neonatal outcomes among women with pregestational diabetes mellitus. National Institute of Child Health and Human Development Network of Maternal-Fetal Medicine Units. Am J Obstet Gynecol 182: 364-369. [Crossref]

7. Sirohiwal D, Dahiya K, Khaneja N (2009) Use of 24-Hour Urinary Protein and Calcium for Prediction of Preeclampsia. Taiwan J Obstet Gynecol 48: 113-115. [Crossref]

8. Kitzmiller JL, Jovanovic L, Brown F, Coustan D, Reader DM (2008) Managing Preexisting Diabetes and Pregnancy: Technical Reviews and Consensus Recommendations for Care. American Diabetes Association, Alexandria,

9. VA Leinonen PJ, Hiilesmaa VK, Kaaja RJ, Teramo KA (2001) Maternal mortality in type 1 diabetes (in Danish). Diabetes Care 24: 1501-1502.

10. Final Recommendation Statement: Low-Dose Aspirin Use for the Prevention of Morbidity and Mortality From Preeclampsia: Preventive Medication. Low-Dose Aspirin Use for the Prevention of Morbidity and Mortality From Preeclampsia: Preventive Medication - US Preventive Services Task Force. https://www. uspreventiveservicestaskforce.org/Page/Document/RecommendationStatementFinal/ low-dose-aspirin-use-for-the-prevention-of-morbidity-and-mortality-frompreeclampsia-preventive-medication. Accessed May 18, 2017.

11. Nageotte MP (2008) Antenatal testing: diabetes mellitus. Semin Perinatol 32: 269-270. [Crossref]

Copyright: (C2018 Gray KM. This is an open-access article distributed under the terms of the Creative Commons Attribution License, which permits unrestricted use, distribution, and reproduction in any medium, provided the original author and source are credited. 